\title{
Los agentes de extracción del cobre. Asociaciones en la fase orgánica ${ }^{(\cdot)}$
}

\author{
F.J. Alguacil $\left.{ }^{*}\right)$
}

\begin{abstract}
Resumen Se describen las asociaciones que presentan algunos agentes de extracción del cobre: hidroxioximas, amidas de ácidos piridincarboxílicos, etc., como consecuencia de interacciones intramoleculares y cuando la fase orgánica contiene, además, componentes orgánicos (modificadores) con átomos de oxígeno en su molécula. Como consecuencia de estas asociaciones cambia la concentración de la especie activa del agente de extracción disminuyendo su efectividad. Se describen algunas de las estructuras de estas asociaciones.
\end{abstract}

Palabras clave: Cobre. Agentes de extracción. Modificadores

\section{Copper extractants. Physicochemical modifications}

\begin{abstract}
The associations of several copper extractants: hydroxyoximes, amides of pyridine carboxilic acids, etc., as a consequence of intramolecular interactions and with various oxygen containing modifiers are described. The various associations of these copper extractants change the active concentration of the extractant and blocks its efectiveness. Some of the structures of such associations are also described.
\end{abstract}

Keywords: Copper. Extractants. Modifiers

\section{INTRODUCCIÓN}

La secuencia de operaciones lixiviación-extracción con disolventes-electroobtención es cada vez más importante en la producción de cobre (1), y está usándose en un número creciente de plantas hidrometalúrgicas que recuperan este metal de los óxidos, las colas de flotación, etc., produciendo el cobre en la forma más económica (2).

Los agentes de extracción empleados para la extracción del cobre de disoluciones acuosas ácidas son las hidroxioximas, que presentan la estructura general que se muestra en la figura 1. En medio amoniacal se han propuesto las B-dicetonas (Fig.2), que a diferencia de las hidroxioximas no coextraen amoniaco; aunque recientemente se ha considerado el empleo de una cetoxima (LIX 84) para su utilización en estas disoluciones alcalinas (1).

En la práctica, se suelen utilizar, tanto cetoximas como aldoximas y mezclas de ambas para la extrac-

\footnotetext{
(•) Trabajo recibido el día 29 de enero de 1998 y aceptado en su forma final el 2 de octubre de 1998.

(*) Centro Nacional de Investigaciones Metalúrgicas, CENIM (CSIC). Av. Gregorio del Amo, 8. 28040Madrid (España).
}

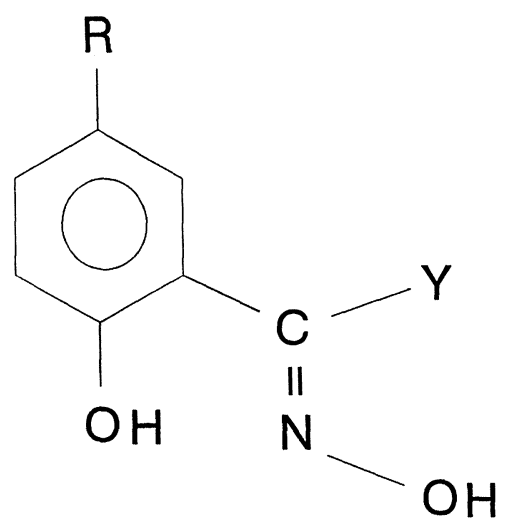

FIG. 1.- Estructura general de las hidroxioximas.

Fig. 1.- General structure of hydroxyoximes.

ción de cobre de disoluciones ácidas (1). Teniendo en cuenta las características estructurales de estos agentes de extracción, todos presentan una cierta tendencia a dar lugar a asociaciones en la fase orgánica; tendencia que no se ha descrito en el caso de las B-dicetonas. En la tabla I se agrupan las hidroxioximas según sus características de trabajo. En un trabajo anterior (1) se dieron los agentes de extracción que, dentro de cada grupo, existen en la actualidad. Las primeras plantas utilizaron el LIX 64N, 


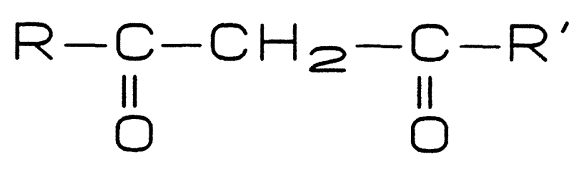

FIG. 2.- Estructura general de las B-dicetonas ( $\mathrm{R}$ y $\mathrm{R}^{\prime}=$ cadenas alquílicas).

FIG. 2.- General structure of $\beta$-diketones ( $R$ y $R^{\prime}=$ alkyl chains).

que contiene, como especie activa, la 2-hidroxi-5nonilbenzofenona oxima ( $\mathrm{Y}=\mathrm{C}_{6} \mathrm{H}_{5}$ en la Fig.1). Para mejorar la extracción del cobre, el producto comercial también contenía LIX 63 (5,8-dietil-7-hidroxi-6-dodecanona oxima).

Cuando comenzaron a desarrollarse estos primeros agentes de extracción se creía que otras oximas, como los derivados de la 2-hidroxi-5-alquilacetofenona oxima ( $\mathrm{Y}=\mathrm{CH}_{3}$ en la Fig.1) o de la 2-hidroxi5-alquilbenzaldehil oxima ( $\mathrm{Y}=\mathrm{H}$ en la Fig.1) se podían hidrolizar en presencia de ácido sulfúrico y, por esa razón, no se podían emplear en la práctica de la extracción del cobre; sin embargo, la realidad mostró que esta hidrólisis es muy lenta y como consecuencia estas oximas se emplearon en la fabricación de nuevos agentes de extracción: SME 529, LIX 84, MOC-45, Acorga P50 y LIX 860.

Todos estos productos son agentes de extracción más fuertes, para el cobre, que el LIX $64 \mathrm{~N}$, por lo tanto, pueden extraer este metal de disoluciones cada vez más ácidas y más diluidas:

$$
\mathrm{Cu}_{\mathrm{ac}}^{2+}+2 \mathrm{HR}_{\mathrm{org}} \Leftrightarrow \mathrm{CuR}_{2} \mathrm{org}+2 \mathrm{H}_{\mathrm{ac}}^{+}
$$

Esta capacidad de extracción no es el único parámetro a considerar; después de la extracción el cobre debe ser reextraido, de la fase orgánica, con ácido sulfúrico de una concentración conveniente y, finalmente, recuperado mediante electroobtención para obtener un cátodo de cobre de una pureza adecuada (por ej. grado A $(99,999 \%$ de pureza)). Por ello, la pureza de este producto final y la cantidad de cobre que se puede transferir en los ciclos de extracción-reextracción son otros de los parámetros a considerar en el proceso.

En el caso de la electroobtención, las celdas trabajan con unos parámetros bien definidos, por ejemplo, una concentración de $\mathrm{H}_{2} \mathrm{SO}_{4}$ de salida de las celdas de 160-180 g/L, que no puede variar; este hecho hace que si el agente de extracción forma un complejo muy estable con el cobre en la fase orgánica, el metal no se reextrae fácilmente y la cantidad de cobre transferida a la fase acuosa de reextracción no es grande. Por lo tanto, para poder aumentar la concentración del cobre reextraido con el electrólito de retorno se hace necesario modificar las propiedades respecto a la extracción de estos reactivos.

Las hidroxioximas, como las representadas en la figura 1, tienen dos grupos activos, uno es el $\mathrm{OH}$ del grupo fenólico, que presenta una acidez baja y otro es el grupo oximino en el que existe un átomo de nitrógeno con un par de electrones libre. Como resultado, se forma un complejo con el cobre que presenta la estructura de la figura 3 (3).

En esta estructura, el grupo hidróxilo de los grupos oximino forma enlaces de hidrógeno intermoleculares con los oxígenos de los grupos fenólicos.

TABLA I.- Comparación de las propiedades de los agentes de extracción basados en cetoximas, aldoximas o mezclas de ambas

TABLE I.- Comparison of properties for extractants based on ketoximes, aldoximes or mixtures

\begin{tabular}{|c|c|c|c|}
\hline Propiedad & Cetoxima & Aldoxima & Mezcla \\
\hline Extracción & Moderada & Muy buena & Según composición \\
\hline Reextracción & Muy buena & Buena & Según composición \\
\hline $\begin{array}{c}\text { Selectividad } \\
\text { Cu-Fe }\end{array}$ & Excelente & Excelente & Excelente \\
\hline Cinética & Muy buena & Excelente & Muy buena \\
\hline Separación fases & Rápida & Rápida & Rápida \\
\hline Estabilidad & Muy buena & Muy buena $\left.{ }^{*}\right)$ & Muy buena \\
\hline Formación lodos & Lenta & Variable & Lenta \\
\hline
\end{tabular}

*) Según el modificador empleado.

(**) Dependiente de la disolución de lixiviación a tratar y del modificador empleado en la fase orgánica. 


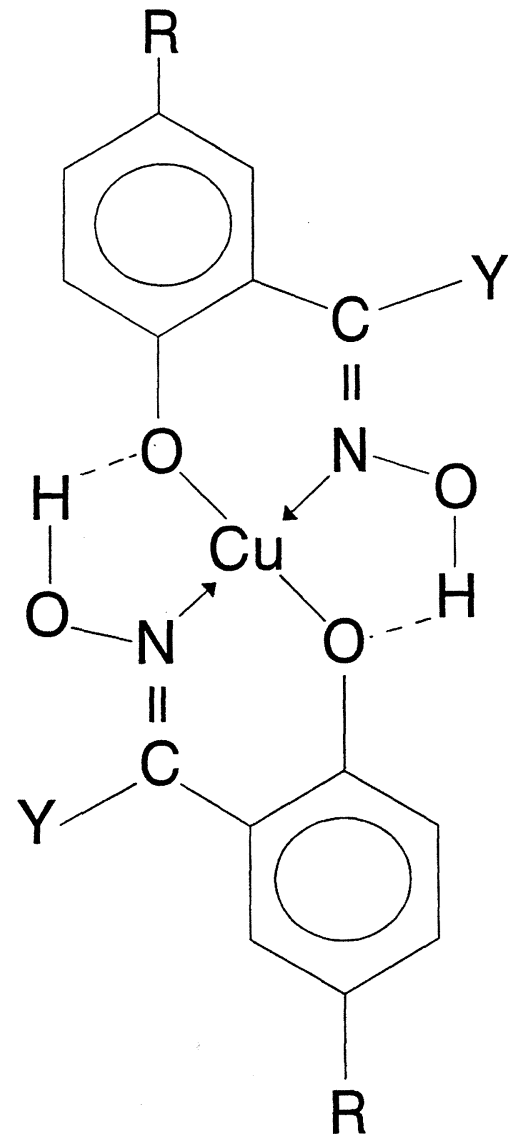

FIG. 3.- Estructura general del complejo formado entre el cobre y las hidroxioximas.

FIG. 3.- General structure of the complex formed by copper and hydroxyoximes.

Este tipo de unión también puede encontrarse en disoluciones de las hidroxioximas, sobre todo si se emplea como diluyente de la fase orgánica algún hidrocarburo que no tenga propiedades de solvatación.

Una estructura monomérica como la que se muestra en la figura 1, con un enlace de hidrógeno intramolecular, se puede encontrar en disoluciones diluidas de la oxima; sin embargo, en disoluciones más concentradas de estos reactivos se pueden formar dímeros (Fig.4) y en este caso los enlaces de hidrógeno intermoleculares adquieren una mayor importancia; por su parte, la ecuación que representa esta dimerización se puede representar mediante el equilibrio:

$$
2 \mathrm{HR}_{\mathrm{org}} \Leftrightarrow(\mathrm{HR})_{2_{\text {org }}}
$$

En las figuras 3 y 4 , los enlaces de hidrógeno no son muy fuertes y se pueden romper, de una forma más o menos fácil, si la fase orgánica contiene otros componentes que pueden formar también enlaces de hidrogéno. La presencia de estos componentes

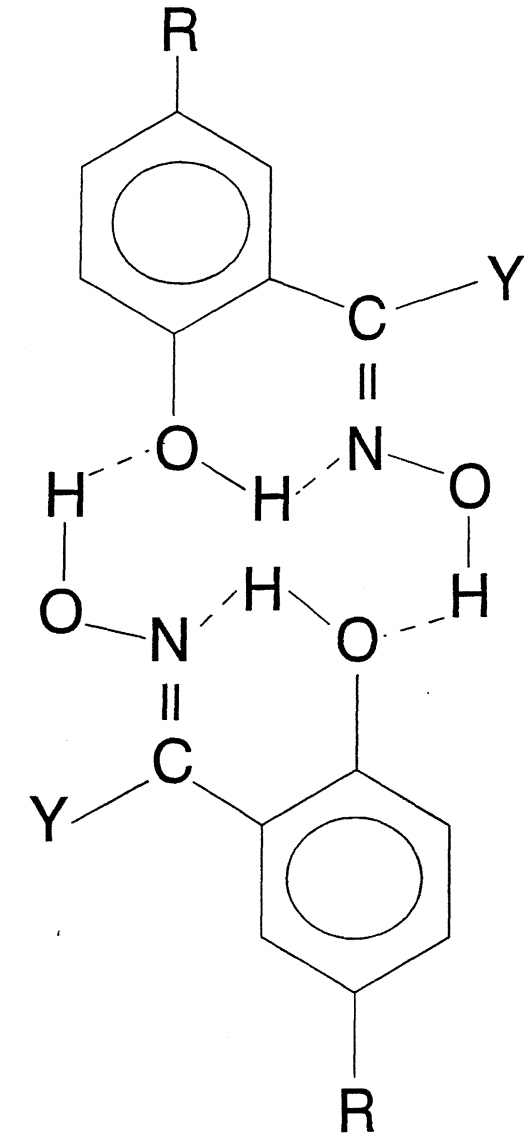

Fig. 4.-Dimerización de las hidroxioximas.

Fig. 4.-Hydroxyoximes dimerization.

altera el equilibrio mostrado en la ec. [2], y, además, puede dar lugar a asociaciones con las moléculas del agente de extracción disminuyendo su concentración efectiva; se entiende que este hecho afecta tanto a la cinética como al equilibrio de extracción del cobre.

Se utiliza esta reactividad para modificar las propiedades de las hidroxioximas comerciales derivadas de la 2-hidroxi-5-alquilbenzaldehil oxima; por ejemplo, en la serie Acorga, LIX 622 y MOC55TD.

Los modificadores deben contener en su molécula grupos capaces de formar enlaces de hidrógeno con estas hidroxioximas; por ejemplo, contener grupos hidroxilo. Desde el principio se emplearon alcoholes y alquilfenoles, de naturaleza hidrofóbica, como modificadores; por ejemplo, nonilfenol en el Acorga P5100 y tridecanol en el MOC-55TD. Estos grupos hidróxilo pueden formar este tipo de enlaces de diversas formas; así, el enlace puede ser a través del hidrógeno ( $\mathrm{ROH}^{\cdots}$ OHoxima) o del oxígeno (RHO ${ }^{\cdots}$ HONoxima). Otros compuestos orgánicos que sólo presentan átomos de oxígeno, como los ésteres, se sumaron posteriormente a los potenciales modificadores de las oximas (Acorga M5640). 
El equilibrio mostrado en la ec. [1] es reversible y se puede emplear para la recuperación del cobre de disoluciones diluidas; por ejemplo, $5 \mathrm{~g} / \mathrm{L}$ del metal, siempre que no presenten una gran concentración de iones cloruro. No son, por lo tanto, agentes de extracción que se pueden emplear, en principio fácilmente, en la recuperación de este metal de disoluciones provenientes de la lixiviación de sulfuros (por ej., con cloruro de hierro(III)) que pueden contener una concentración elevada de cobre y en este medio clorurado. El proceso CUPREX se desarrolló para recuperar cobre de estas disoluciones (4). En este proceso, la etapa de extracción del metal está basada en el empleo de un agente de extracción de tipo piridínico que presenta un carácter solvatante; el agente de extracción es el Acorga CLX 50, que tiene al diisodecil piridin-3,5-dicarboxilato como grupo activo (Fig.5).

Posteriormente, a este se han propuesto otros ésteres o amidas de ácidos piridincarboxílicos como agentes de extracción para el cobre y otros metales (5-12). Todos estos compuestos se pueden protonar y la transferencia de las especies metálicas puede deberse tanto a procesos de solvatación como de intercambio aniónico:

$$
\begin{gathered}
\mathrm{CuCl}_{2_{\mathrm{ac}}}+2 \mathrm{~L}_{\text {org }} \Leftrightarrow \mathrm{CuCl}_{2 \mathrm{~L}_{2} \text { org }} \\
\mathrm{CuCl}_{4_{\mathrm{ac}}}^{2-}+2 \mathrm{HL}^{+} \mathrm{Cl}_{\text {org }}^{-} \Leftrightarrow \mathrm{CuCl}_{4}(\mathrm{HL})_{2_{\text {org }}}+2 \mathrm{Cl}_{\mathrm{ac}}^{-}
\end{gathered}
$$

La ecuación [4] no es específica del cobre y por lo tanto la selectividad en la extracción puede no ser alta. En el Acorga CLX50 se eligió el grupo activo descrito anteriormente para disminuir la posibilidad de protonación del agente de extracción y, además, permitir la extracción del cobre de un medio $3-5 \mathrm{M}$ en cloruro. El empleo de algunos de los ácidos piridincarboxílicos puede encontrar dificultades en la etapa de reextracción del cobre, que en estos casos se hace con disoluciones con una fuerza iónica baja.

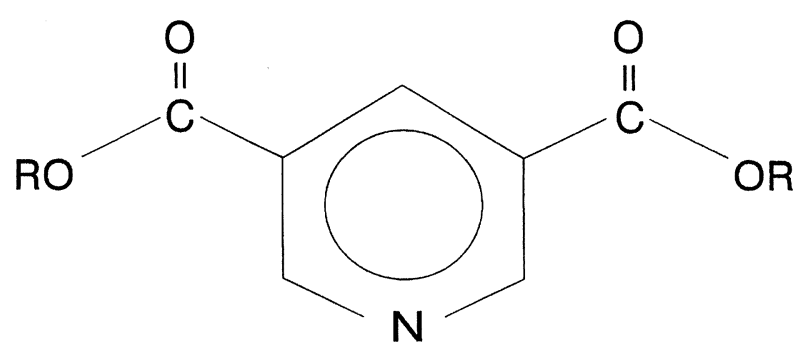

FIG. 5.- Estructura general del componente activo del Acorga CLX50.

FIG. 5.- General structure of the active component of Acorga CLX50.
Debido a su estructura, estos compuestos no se pueden asociar en las fases orgánicas, aunque pueden formar algún tipo de asociación con grupos donadores de electrones; por ejemplo, alcoholes que estén presentes en las disoluciones orgánicas.

Se ha propuesto un nuevo proceso (Henkel) que se basa en el empleo de un sistema mixto de agentes de extracción (13-15), y que consta de varias etapas de procesamiento. En la primera etapa, el cobre se extrae, en medio cloruro, mediante un agente de extracción del estilo del Acorga CLX50; la reacción es similar a la mostrada en la ec. [3]; seguidamente, la fase orgánica se lava con una fase acuosa de $\mathrm{pH}$ adecuado (alcalino); como la fase orgánica contiene, además, un agente de extracción tipo quelato (hidroxioximas o ß-dicetonas), en esta etapa el cobre forma el complejo correspondiente con este reactivo tipo quelato y a la vez se transfieren los iones cloruro al medio acuoso:

$$
\begin{aligned}
2 \mathrm{HR}_{\text {org }}+ & \mathrm{CuCl}_{2} \mathrm{~L}_{2_{\text {org }}}+2 \mathrm{NH}_{3_{\mathrm{ac}}} \Leftrightarrow \mathrm{CuR}_{2_{\text {org }}}+ \\
& +2 \mathrm{~L}_{\text {org }}+2 \mathrm{NH}_{4} \mathrm{Cl}_{\mathrm{ac}}
\end{aligned}
$$

Después de esta etapa de lavado, el cobre puede reextraerse de forma convencional (ec. [1]) y recuperado mediante electroobtención. El proceso se puede complicar ya que el agente neutro puede protonarse:

$$
\mathrm{H}_{\mathrm{ac}}^{+}+\mathrm{L}_{\mathrm{org}} \Leftrightarrow \mathrm{HL}_{\mathrm{org}}^{+}
$$

con lo que debe reconvertirse a la forma neutra $\mathrm{o}$ clorurada.

En algunos casos se considera el empleo de mezclas de agentes de extracción para formar enlaces intermoleculares y así lograr mezclas de estos con propiedades variadas (1); por ejemplo, en el LIX 984 y MOC-80TD. En estos casos, el reactivo más débil actúa como modificador formando enlaces hidrógeno con las moléculas de la especie activa considerada como más fuerte.

\section{INTRAASOCIACIÓN DE LOS AGENTES DE EXTRACCIÓN}

Se sabe que las hidroxioximas diluidas en diluyentes orgánicos se pueden asociar. Las hidroxioximas alifáticas (LIX 63) forman asociaciones lineales mientras que las hidroxioximas aromáticas forman dímeros cíclicos como el que se muestra en la figura 4 (16).

El grado de asociación es función de diversos factores: tipo de diluyente, tipo de oxima y concentración de esta en la fase orgánica. En el caso de emplear diluyentes no solvatantes, la asociación es 
mayor que en el caso de utilizar diluyentes con una cierta capacidad de solvatación. En general, las oximas alifáticas forman mayores asociaciones que las aromáticas (Fig.1). No se encuentran diferencias en los espectros infrarrojos de distintas oximas aromáticas; tanto los derivados de la 2-hidroxi-5-alquilbenzaldehil oxima como de la 2-hidroxi-5-alquilacetofenona oxima existen sólo como el isómero $\mathrm{E}$ (Fig.1); sin embargo, los derivados de la 2-hidroxi5 -alquilbenzofenona oxima pueden formar los isómeros E y Z (Fig.6).

En el caso del isómero Z, el espectro IR muestra diferencias respecto al isómero $\mathrm{E}$. En este último aparecen bandas a $3.220,3.420$ y $3.580 \mathrm{~cm}^{-1}$, la primera se atribuye al grupo $\mathrm{OH}$ fenólico que está fuertemente enlazado con el nitrógeno del grupo oxima (Fig.4), las bandas a 3.420 y $3.580 \mathrm{~cm}^{-1}$ se atribuyen a los grupos $\mathrm{N}-\mathrm{OH}$ asociados y monoméricos.

El grado de asociación de las oximas depende de la concentración de estas en las fases orgánicas. En general, la dimerización de las oximas puede no tenerse en cuenta en disoluciones que contienen hidrocarburos alifáticos o aromáticos si las concetraciones de estas son menores de $0,01 \mathrm{M}$ y $0,1 \mathrm{M}$ respectivamente. En cualquier caso, las constantes de dimerización presentan valores de pocas unidades (diluyentes aromáticos) o varían entre 10-200 $\mathrm{dm}^{3} / \mathrm{mol}$ (diluyentes alifáticos), valores muy bajos si se comparan con los obtenidos para diversos derivados organosfosforados que presentan constantes de dimerización del orden de $10^{4}$.

Esto hace que la asociación de las hidroxioximas pueda destruirse en presencia de otros componentes (por ej., alcoholes) que tiendan a formar enlaces

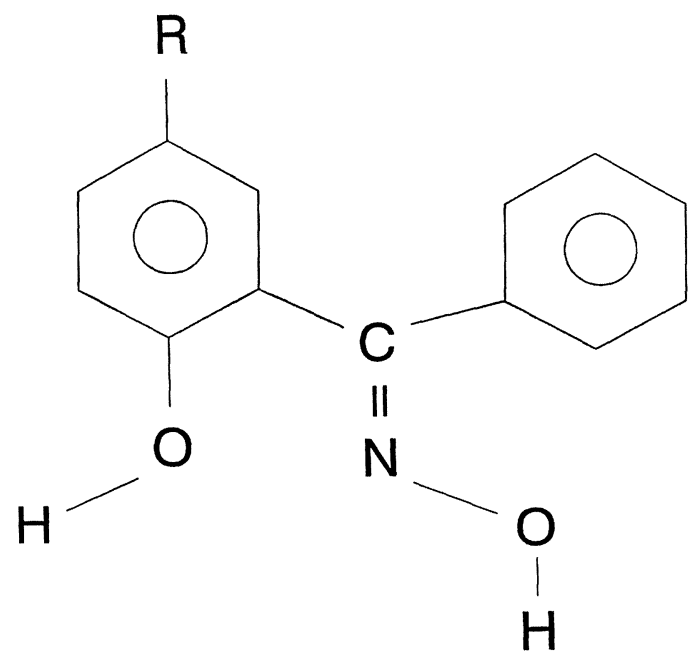

FIG. 6.- Estructura general del isómero $\mathrm{Z}$ de las benzofenona oximas.

FIG. 6.- General structure of Z-isomer of benzophenone oximes. con estas moléculas. El agua, si está presente de alguna manera en la fase orgánica, puede actuar de la misma forma, disminuyendo la asociación de las oximas. Existe otro factor que debe ser considerado al tener en cuenta la posibilidad de asociación en las oximas, tanto el grupo hidroxilo como el oximino presentan una baja acidez e hidrofilidad; por esto, las oximas con estructuras como la representada en la figura 1, no forman micelas inversas ni microemulsiones como los derivados organofosforados ácidos o los ácidos sulfónicos (17).

Como ya se ha mencionado, los derivados piridínicos (Fig.5) al no presentar en su estructura átomos de hidrógeno lábiles, no pueden dar lugar a asociaciones intramoleculares.

\section{INTRAASOCIACIÓN DE LOS MODIFICADORES}

Tanto los alcoholes como los alquilfenoles pueden actuar como donadores de electrones (a través del átomo dé oxígeno) o receptores de electrones (vía hidrógeno), por lo que pueden asociarse en diluyentes no polares. Los espectros infrarrojos muestran las posibles diferencias de estas asociaciones, así a $3.650 \mathrm{~cm}^{-1}$ aparece una banda estrecha que se atribuye a las especies monómeras, mientras que a $3.525 \mathrm{~cm}^{-1}$ aparece una banda ancha característica de estas asociaciones. Algunos alcoholes pueden formar hasta tetrámeros, que pueden ser lineales o cíclicos (Figs. 7 y 8 respectivamente); sin embargo, si aumentan las ramificaciones de las cadenas alquílicas existe una mayor probabilidad de que el alcohol no se asocie. En general, los alcoholes con cadenas lineales pueden actuar como modificadores mientras que los más ramificados no son activos.

La asociación de los alcoholes depende tanto de la estructura del mismo como de su peso molecular (18 y 19). Los dímeros son siempre lineales, en cambio en las asociaciones mayores, las estructuras pueden ser lineales o cíclicas. En las figuras 9 a 12 se muestran algunas de estas estructuras para asociaciones de n-butanol y/o t-butanol.

Se debe, por lo tanto, tener en consideración estas asociaciones, que por otra parte se pueden relacionar mediante el siguiente esquema de reacciones:

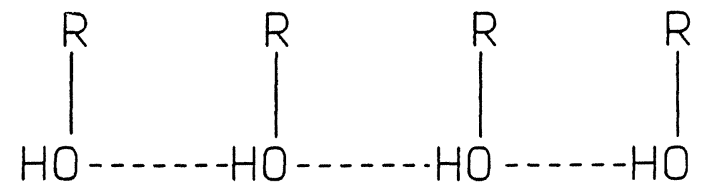

FIG. 7.- Tetrámero lineal de los alcoholes.

FIG. 7. - Lineal tetramer of alcohols. 


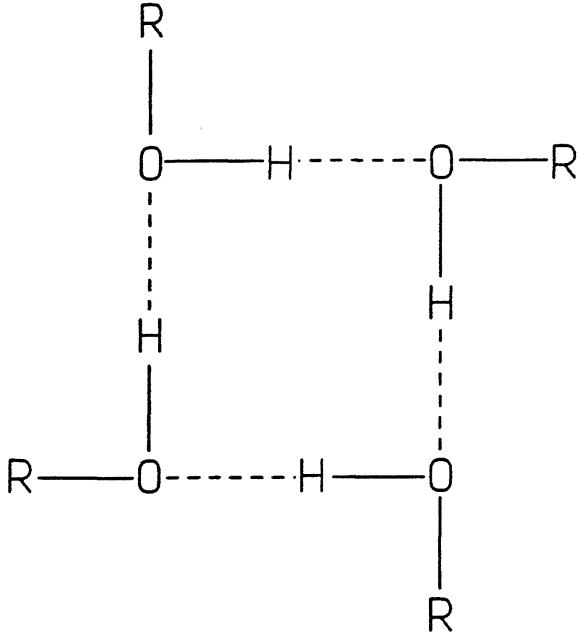

Fig. 8.- Tetrámero cíclico de los alcoholes.

FIG. 8.- Cyclic tetramer of alcohols.

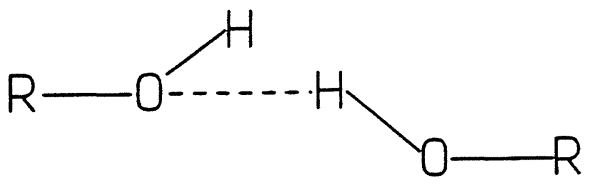

FIG. 9.- Dímero lineal en el n-butanol $\left(\mathrm{R}=\mathrm{C}_{4} \mathrm{H}_{9}\right)$.

FIG. 9.-Lineal dimer in $n$-butanol $\left(R=\mathrm{C}_{4} H_{9}\right)$.

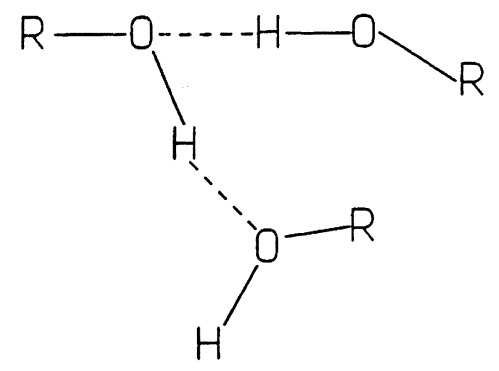

FIG. 10.- Trímero lineal en el n-butanol $\left(\mathrm{R}=\mathrm{C}_{4} \mathrm{H}_{9}\right)$.

FIG. 10. - Lineal trimer in n-butanol $\left(R=C_{4} H_{9}\right)$.

$$
\begin{gathered}
2 \mathrm{ROH}=(\mathrm{ROH})_{2} \text { (lineal) } \\
(\mathrm{ROH})_{2}(\text { lineal })+\mathrm{ROH}=(\mathrm{ROH})_{3} \text { (lineal) } \\
(\mathrm{ROH})_{3} \text { (lineal) }=(\mathrm{ROH})_{3} \text { (cíclico) } \\
(\mathrm{ROH})_{3}(\text { lineal })+\mathrm{ROH}=(\mathrm{ROH})_{4} \text { (lineal) } \\
(\mathrm{ROH})_{4}(\text { lineal })=(\mathrm{ROH})_{4} \text { (cíclico) }
\end{gathered}
$$

Los fenoles tienden a formar asociaciones mayores que los alcoholes y estas son lineales. La tendencia a formar estas asociaciones hace que por el contrario no sea tan fácil su asociación con las hidroxioximas (20).

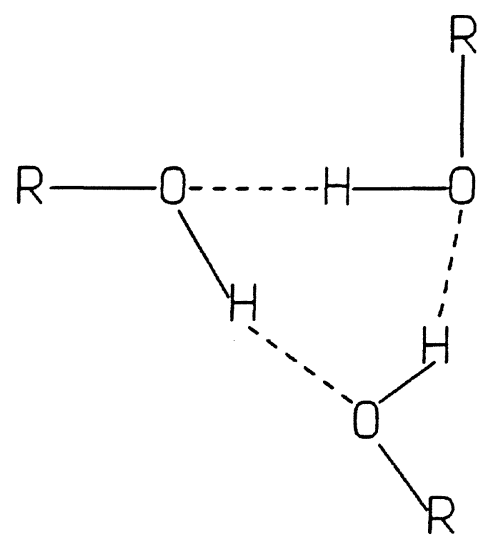

FIG. 11.- Trímero cíclico en el t-butanol $\left(\mathrm{R}=\mathrm{C}_{4} \mathrm{H}_{9}\right)$.

FIG. 11.-Cyclic trimer in t-butanol $\left(R=C_{4} H_{9}\right)$.

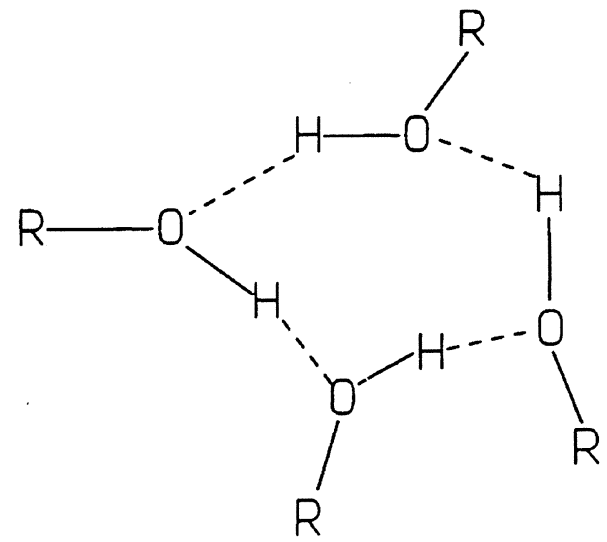

FIG. 12.- Tetrámero cíclico en el t-butanol $\left(\mathrm{R}=\mathrm{C}_{4} \mathrm{H}_{9}\right)$.

FIG. 12.-Cyclic tetramer in t-butanol $\left(R=C_{4} H_{9}\right)$.

\section{INTERASOCIACIÓN DE LAS HIDROXIOXIMAS CON LOS MODIFICADORES}

Las hidroxioximas forman asociaciones 1:1 y 1:2 con los alcoholes (21), no estando bien definida cuál es su estructura. Los alcoholes pueden aceptar electrones del átomo de oxígeno de los grupos fenólico u oximino de la oxima, aunque también pueden ceder electrones al hidrógeno del grupo oximino del agente de extracción. La predominancia de estas especies depende de la estructura del alcohol.

Los alcoholes que presentan una estructura muy poco ramificada actúan como receptores de electrones para formar enlaces de hidrógeno con los átomos de oxígeno de los grupos fenólico u oximino. Los alcoholes que presentan estructuras ramificadas son donadores de electrones para formar enlaces de hidrógeno con el hidrógeno del grupo oximino. Este hecho se debe probablemente a un 
efecto estérico. Algunas estructuras de estas asociaciones 1:1 entre una salicilaldoxima y el n-butanol o t-butanol se muestran en las figuras 13 y 14 .

La figura 15 muestra la estructura para una asociación 1:2 (oxima:alcohol).

Los alquilfenoles también pueden formar asociaciones 1:1 y 1:2 con las oximas. Estos alquilfenoles también pueden actuar como receptores de electrones en las asociaciones 1:1, presentando los compuestos una estructura similar a la que se ha mostrado en la figura 13.

Existen otros compuestos que contienen átomos de oxígeno que pueden emplearse para modificar las hidroxioximas; entre estos compuestos se encuentran las cetonas, los ésteres y los éteres. Como estos compuestos sólo pueden actuar como donadores de electrones para formar enlaces de hidrógeno con el átomo de hidrógeno del grupo oximino, sólo se pueden formar las estructuras 1:1. Las figuras 16 y 17 muestran estas estructuras para el caso de asociación de la salicilaldoxima con la 2hexanona y el acetato de butilo respectivamente. La capacidad de asociación de estos componentes de la fase orgánica disminuye en el orden: cetonas > ésteres > éteres.

\section{ASOCIACIONES ENTRE DERIVADOS DE ÁCIDOS PIRIDINCARBOXÍLICOS Y ALCOHOLES}

Los ésteres de los ácidos piridincarboxílicos, como el componente activo del Acorga CLX50 (Fig.5) presentan tres clases de átomos donadores de electrones:

- los átomos de oxígeno de los grupos carbonilo $(\mathrm{C}=\mathrm{O})$

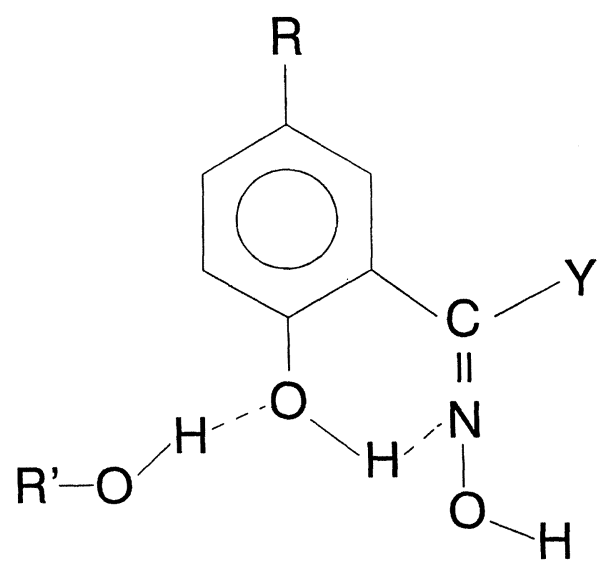

FIG. 13.- Asociación entre una salicilaldoxima y el n-butanol $\left(\mathrm{R}^{\prime}=\mathrm{C}_{4} \mathrm{H}_{9}\right)$. Complejo 1:1.

FIG. 13.-Association between salicylaldoxime and $n$-butanol $\left(R^{\prime}=C_{4} H_{9}\right)$. 1:1 complex.

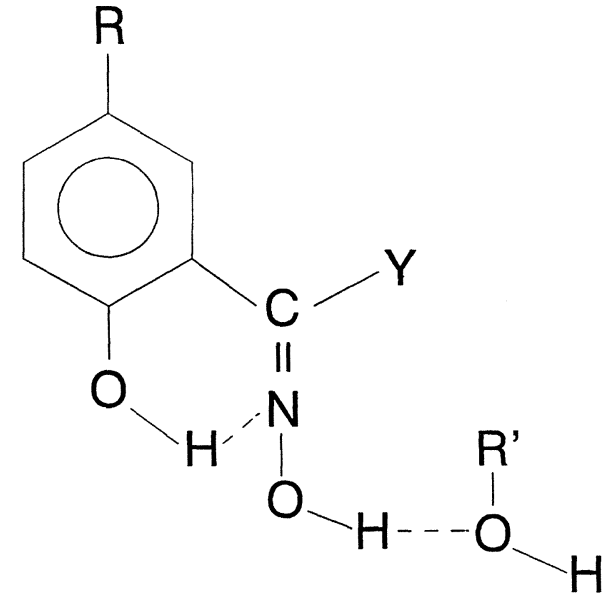

FIG. 14.- Asociación entre una salicilaldoxima y el t-butanol $\left(\mathrm{R}^{\prime}=\mathrm{C}_{4} \mathrm{H}_{9}\right)$. Complejo 1:1.

FIG. 14.- Association between salicylaldoxime and t-butanol $\left(R^{\prime}=C_{4} H_{9}\right)$. 1:1 complex.

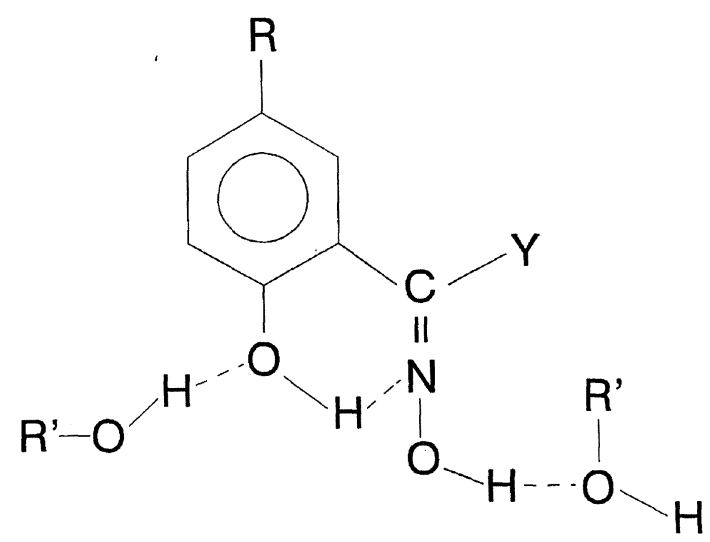

FIG. 15.- Asociación entre una salicilaldoxima y el n-butanol $\left(\mathrm{R}^{\prime}=\mathrm{C}_{4} \mathrm{H}_{9}\right)$. Complejo 1:2.

FIG. 15.-Association between salicylaldoxime and $n$-butanol $\left(R^{\prime}=C_{4} H_{9}\right)$. 1:2 complex.

- los átomos de oxígeno de los grupos alcoxi (RO) del éster,

- el átomo de nitrógeno, aunque en este caso la capacidad de donación de los electrones disminuye por el efecto de los grupos éster que tienden a retirar carga.

Las amidas correspondientes (Fig. 18) contienen otros átomos de nitrógeno aparte del piridínico; todos estos átomos pueden formar enlaces de hidrógeno con los modificadores. Sin embargo, existe una diferencia fundamental respecto a las hidroxioximas, los derivados piridínicos extraen el cobre vía una interacción con el átomo de nitrógeno piridínico, y la asociación con los alcoholes se lleva a cabo a través de átomos (oxígeno y/o nitrógeno) que no interaccionan con el cobre; así, en el caso de 


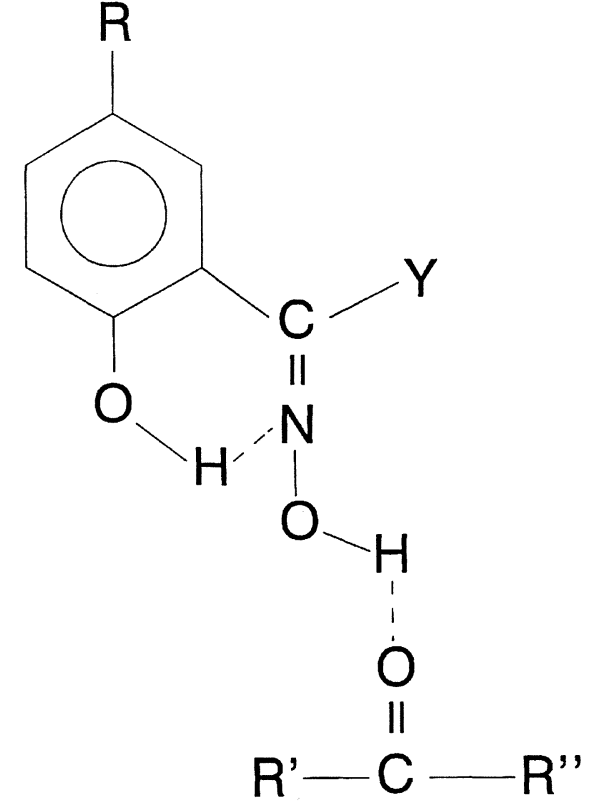

FIG. 16.- Asociación entre una salicilaldoxima y la 2-hexanona $\left(\mathrm{R}^{\prime}=\mathrm{CH}_{3}, \mathrm{R}^{\prime \prime}=\mathrm{C}_{4} \mathrm{H}_{12}\right)$.

FIG. 16.- Association between salicylaldoxime and 2-hexanone $\left(\mathrm{R}^{\prime}=\mathrm{CH}_{3}, \mathrm{R}^{\prime \prime}=\mathrm{C}_{4} \mathrm{H}_{12}\right)$.

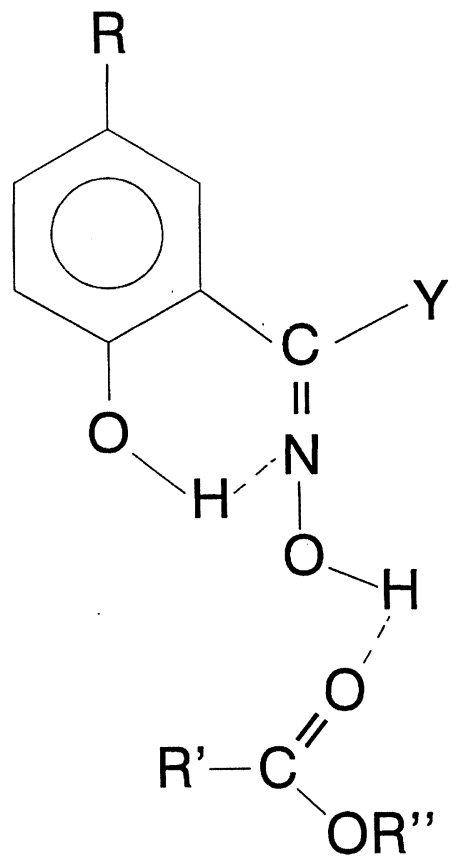

FIG. 17.- Asociación entre una salicilaldoxima y el acetato de butilo $\left(\mathrm{R}^{\prime}=\mathrm{CH}_{3}, \mathrm{R}^{\prime \prime}=\mathrm{C}_{4} \mathrm{H}_{9}\right)$.

FIG. 17.-Association between salycilaldoxime and butyl acetate $\left(\mathrm{R}^{\prime}=\mathrm{CH}_{3}, \mathrm{R}^{\prime \prime}=\mathrm{C}_{4} \mathrm{H}_{9}\right)$.

producirse estas asociaciones su efecto es mucho menor, respecto a la extracción del $\mathrm{Cu}^{2+}$, que con las hidroxioximas.

Los alquil-piridin-monocarboxilatos, cuya estructura general se muestra en la figura 19, pue-

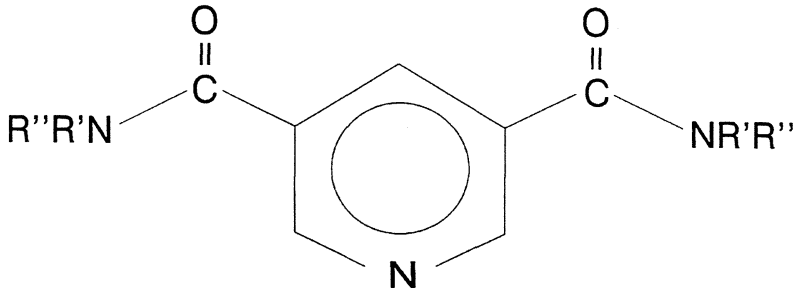

FIG. 18.- Estructura general de una amida de un ácido piridincarboxílico.

FIG. 18.- General structure of an amide of pyridincarboxylic acid.

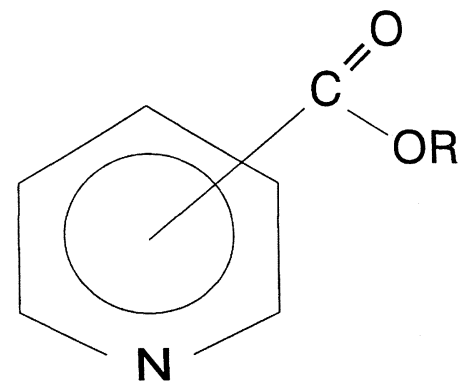

FIG. 19.- Estructura general de los alquil-piridinmonocarboxilatos.

FIG. 19.- General structure of alkyl pyridine monocarboxylates.

den presentar el grupo éster en las posiciones 2, 3 ó 4; en todos los casos se forman asociaciones 1:1 con los alcoholes, siendo también posible en algunos casos asociaciones 1:2 (22).

\section{CONCLUSIONES}

Los agentes de extracción del cobre, especialmente los derivados de la oxima, pueden ver modificadas sus propiedades, respecto a la extracción de este metal, por distintas asociaciones inter y/o intramoleculares. Aunque la extracción de cobre se puede representar por el equilibrio general mostrado en la ec. [1], dependiendo de las condiciones experimentales se puede necesitar tener en cuenta otros equilibrios:

a) asociación de la oxima:

$$
2 \mathrm{HR}_{\mathrm{org}} \Leftrightarrow(\mathrm{HR})_{2_{\mathrm{org}}}
$$

b) asociación de la oxima con el modificador $(\mathrm{n}=$ 1,2):

$$
\mathrm{HR}_{\text {org }}+\mathrm{nROH}_{\text {org }} \Leftrightarrow \mathrm{HR} \cdot \mathrm{nROH}_{\mathrm{org}}
$$

c) asociación del modificador $(m=3,4)$ : 


$$
\mathrm{mROH}_{\mathrm{org}} \Leftrightarrow(\mathrm{ROH})_{\mathrm{m}_{\mathrm{org}}}
$$

En general, el efecto de la ecuación [12] sobre el equilibrio de la ecuación [1] puede ser despreciado, teniendo una mayor influencia sobre este último los equilibrios representados por las ecuaciones [13] y [14].

En el caso de los derivados de los ácidos piridincarboxílicos, aunque se pueden producir asociaciones intermoleculares, estas no parecen afectar apreciablemente a la extracción del cobre por estos agentes de extracción.

\section{Agradecimiento}

Al CSIC por la ayuda prestada para la realización del trabajo.

\section{REFERENCIAS}

(1) Alguacil, F.J. Rev. Metal. Madrid, 34, 1998: 499-506.

(2) Szymanowski, J. Hydroxyoximes and Copper Hydrometallurgy, CRC Press, Boca Raton (EE.UU.), 1993.

(3) Borowiak-Resterna, A. y Szymanowski, J.J. Radioanal. Nucl. Chem., 150, 1991: 235.

(4) Dalton, R.F., Diaz, G., Hermana, E., Price, R. y Zunkel, A.D. Copper'91/Cobre 91, W.G. Cooper, D.J. Kemp, G.E. Lagos y K.G. Tan (Eds.), Pergamon Press, Nueva York, 1991: 61.

(5) Szymanowski, J., Jakubiak, A., Cote, G., Bauer, D. y Beger, J. Solvent Extraction in the Process Industries, D.H. Logsdail y M.J. Slater (Eds.), SCI/Elsevier, London (RU), 1993: 1311.

(6) Cote, G., Jakubiak, A., Bauer, D., Szymanowski, J., Mokili, B. y Poitrenaud, C. Solvent Extr. Ion Exch., 12, 1994: 99.
(7) Szymanowski, J., Cote, G., Sobczynska, A., Firgolski, K. y JaKubiaK, A. Solvent Extr. Ion Exch., 12, 1994: 69.

(8) Jakubiak, A., Cote, G. y Szymanowski, J. Value Adding Through Solvent Extraction, D.C. Shallcross, R. Paimin y L.M. Prvcis (Eds.), UM, Melbourne, 1996: 517.

(9) Szymanowski, J., Jakubiak, A., Cote, G., Bauer, D. y CierPisZewski, R. Hydrometallurgy'94, SCI/Chapman and Hall, London (RU), 1994: 666.

(10) Borowiak-Resterna, A., Szymanowski, J., Cierpiszewski, R., Prochaska, K. y BanczyK, I. Solvent extraction in the Process Industries, D.H. Logsdail y M.J. Slater (Eds.), SCI/Elsevier, London (RU), 1994: 578.

(11) Borowiak-Resterna, A. Solvent Extr. Ion Exch., 12, 1994: 557.

(12) Borowiak-Resterna, A. y Szymanowski, J. Value Adding Through Solvent Extraction, D.C.Shallcross, R.Paimin y L.M.Prvcis (Eds.), UM, Melbourne, 1996: 569.

(13) Kyuchoukov, G. y Minaylov, I. Hydrometallurgy, 27, 1991: 27.

(14) Kyuchoukov, G. y Mishonov, I. Solvent Extr. Ion Exch., 11, 1993: 555.

(15) Kyuchoúkov, G. y Mishonov, I. Hydrometallurgy, 41, 1996: 89.

(16) Szymanowski, J., Alejski, K. y Prochaska, K. Hydrometallurgy, 25, 1990: 329.

(17) Szymanowski, J., Cote, G., Blondet, I., Bouvier, C., BAUER, D. y SABOT, J.L. Hydrometallurgy, 44, 1997: 163.

(18) Bogacki, M.B. y Szymanowski, J. Solvent Extr. Res. Dev. Jpn., 3, 1996: 10.

(19) Bogacki, M.B. y Szymanowski, J. Value Adding Through Solvent Extraction, D.C. Shallcross, R. Paimin y L.M. Prvcis (Eds.), UM, Melbourne, 1996: 383.

(20) Barela, R. y Buchowski, H. Fluid Phase Equilibria, 59, 1990: 99.

(21) Bogacki, M.B., Lozynski, M. y Szymanowski, J. Solvent Extr. Res. Dev. Jpn., 1, 1994: 108.

(22) Bogacki, M.B. y Szymanowski, J. Solvent Extr. Ion Exch., 14, 1996: 897. 